\title{
Cooling of a Compact Star with a LOFF Matter Core
}

\author{
Roberto Anglan:* \\ Università di Bari, I-70126 Bari, Italia and \\ I.N.F.N., Sezione di Bari, I-70126 Bari, Italia
}

(Dated: July 11, 2018)

\begin{abstract}
Specific heat and neutrino emissivity due to direct URCA processes for quark matter in the color superconductive Larkin-Ovchinnikov-Fulde-Ferrell (LOFF) phase of Quantum-Chromodynamics have been evaluated. The cooling rate of simplified models of compact stars with a LOFF matter core is estimated.

PACS numbers: 12.38.-t, 26.60.+c, 97.60.Jd
\end{abstract}

Emission of $\beta$-neutrino due to direct URCA processes is the dominant cooling mechanism for an aged compact star 1]. In the core of a neutron star, where hadronic density is large enough to produce [2] deconfined quark matter, direct URCA processes can take place [3]. At density and temperature relevant for aged pulsars, quark matter could be in one of the possible color superconducting (CSC) phases [4]. The ground state of a color and electrically neutral matter of deconfined quarks in weak equilibrium is still a matter of debate [5]. A phase named LOFF which is energetically favored with respect to the normal quark matter and gapless phases [6] in a wide range of densities, has attracted theoretical attention [7]. This phase turns out to be also chromomagnetically stable [8]. The LOFF pairing is characterized by a non vanishing total momentum of the pair. The one-plane wave structure ansatz, defined by

$$
\left\langle\psi_{\alpha i}(x) C \gamma_{5} \psi_{\beta j}(x)\right\rangle \propto \sum_{I=1}^{3} \Delta_{I} e^{2 i \mathbf{q}_{I} \cdot \mathbf{r}} \epsilon_{\alpha \beta I} \epsilon_{i j I}
$$

$(i, j=1,2,3$ flavor indices, $\alpha, \beta=1,2,3$ color indices) has been considered in Ref. [9] and found energetically favored with respect to the gapless and the unpaired phases in a certain range of values of $\delta \mu$. In Eq. (1), $2 \mathbf{q}_{I}$ represents the momentum of the Cooper pair and the gap parameters $\Delta_{1}, \Delta_{2}, \Delta_{3}$ describe respectively $d-s, u-s$ and $u-d$ pairing. For sufficiently large $\mu$ the energetically favored phase is characterized by $\Delta_{1}=0, \Delta_{2}=\Delta_{3}$ and $\mathbf{q}_{2}=\mathbf{q}_{3}$.

The presence of quark matter in the LOFF state in the core of a pulsar will affect the neutrino emissivity and consequently the cooling process. Neutrino emissivity is defined as the energy loss by $\beta$-decay per volume unit per time unit. We have discussed these effects by comparing various models of stars similarly to the analysis performed in Ref. [10] for the gCFL phase (for similar calculations see Ref. [1]]). We have used 12] a simplified approach based on the study of three different toy models of stars. The first model (denoted as I) is a star consisting of noninteracting "nuclear" matter (neu-

*Electronic address: roberto.anglani@ba.infn.it trons, protons and electrons) with mass $M=1.4 M_{\odot}$, radius $R=12 \mathrm{~km}$ and uniform density $n=1.5 n_{0}$, where $n_{0}=0.16 \mathrm{fm}^{-3}$ is the nuclear equilibrium density. The nuclear matter is assumed to be electrically neutral and in beta equilibrium. The second model (II) is a star containing a core of radius $R_{1}=5 \mathrm{~km}$ of neutral unpaired quark matter at $\mu=500 \mathrm{MeV}$, with a mantle of noninteracting nuclear matter with uniform density $n$. Solution of the Tolman-Oppenheimer-Volkov equations gives a mass-radius relation so that a mass $M=1.4 M_{\odot}$ corresponds to a star radius $R_{2}=10 \mathrm{~km}$. The model III is represented by a compact star containing a core of electric and color neutral three flavors quark matter in the LOFF phase, with $\mu=500 \mathrm{MeV}$ and $m_{s}^{2} / \mu=140 \mathrm{MeV}$.

The main processes of cooling are dominated by neutrino emission in the early stage of the lifetime of the pulsar and by photon emission at later ages. The cooling rate is governed by the following differential equation:

$$
\begin{aligned}
\frac{d T}{d t} & =-\frac{L_{\nu}+L_{\gamma}}{V_{n m} c_{V}^{n m}+V_{q m} c_{V}^{q m}} \\
& =-\frac{V_{n m} \varepsilon_{\nu}^{n m}+V_{q m} \varepsilon_{\nu}^{q m}+L_{\gamma}}{V_{n m} c_{V}^{n m}+V_{q m} c_{V}^{q m}} .
\end{aligned}
$$

Here $T$ is the inner temperature at time $t ; L_{\nu}$ and $L_{\gamma}$ are neutrino and photon luminosities, i.e. emissivity by the corresponding volume. The superscripts $n m$ and $q m$ refer, respectively, to nuclear matter and quark matter including the superconductive phase; $c_{V}^{n m}$ and $c_{V}^{q m}$ denote specific heats of the two forms of hadronic matter. Eq. (2) is solved imposing a given temperature $T_{0}$ at a fixed early time $t_{0}$ (we use $T_{0} \rightarrow \infty$ for $t_{0} \rightarrow 0$ ). More detailed results are reported in Ref. 12. In Fig. 11 we can see the star surface temperature as a function of time. Solid line (black online) is for model I; dashed curve (red online) refers to model II; the dotted line (blue online) is for model III and it is obtained for the following values of the parameters: $\mu=500 \mathrm{MeV}, m_{s}^{2} / \mu=140 \mathrm{MeV}$, $\Delta_{1}=0, \Delta_{2}=\Delta_{3} \simeq 6 \mathrm{MeV}$, where $m_{s}$ is the strange quark mass. Let us observe that the neutrino emissivity decreases at increasing values of $m_{s}$. This is due to the fact that $\Delta$ decreases as one approaches the second order phase transition to the normal state. At the same time the quark matter tends to a normal Fermi liquid state, but in this case the description of Iwamoto which includes 


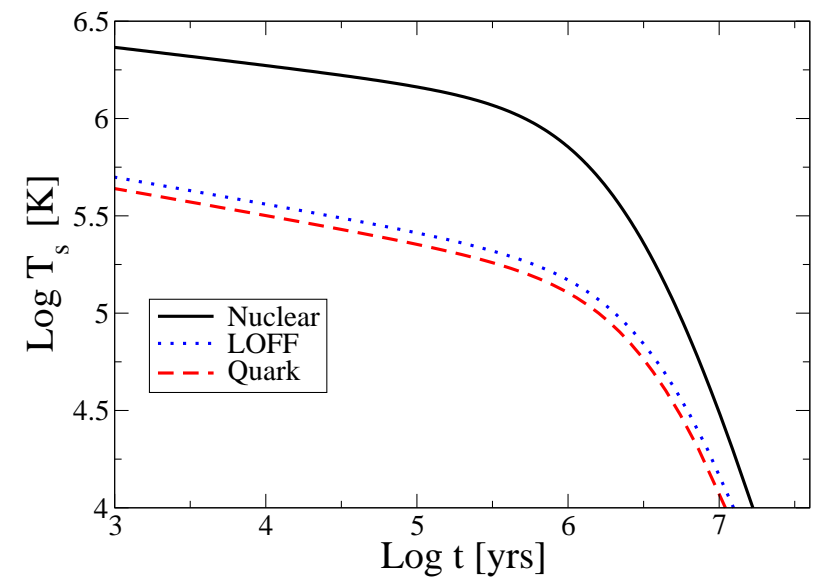

FIG. 1: (Color online) Surface temperature $T_{s}$, in Kelvin, as a function of time, in years, for the three toy models of pulsars described. Solid black curve refers to a neutron star formed by nuclear matter with uniform density $n=0.24 \mathrm{fm}^{-3}$ and radius $R=12 \mathrm{Km}$ (model I); dashed line (red online) refers to a star with $R_{2}=10 \mathrm{~km}$, having a mantle of nuclear matter and a core of radius $R_{1}=5 \mathrm{Km}$ of unpaired quark matter, interacting via gluon exchange (model II); dotted curve (blue online) refers to a star like model II, but in the core there is quark matter in the LOFF state; see 12] for more details. All stars have $M=1.4 M_{\odot}$.

Fermi liquid effects and one gluon exchange, must be adopted. For unpaired quark matter we use $\alpha_{s} \simeq 1$, the value corresponding to $\mu=500 \mathrm{MeV}$ and $\Lambda_{\mathrm{QCD}}=250$ $\mathrm{MeV}$. The use of perturbative QCD at such small momentum scales is however questionable. Therefore the results for model II should be considered with some caution and the curve is plotted only to allow a comparison with the other models. In any case it is important to remark that the apparent similarity between the LOFF curve and the unpaired quark curve depends on the fact that the LOFF phase is gapless. This yields a parametric dependence on temperature analogous to that of the unpaired quark matter: $c_{V} \sim T$ and $\varepsilon_{\nu} \sim T^{6}$. How- ever the similarity between the curves of models II and III should be considered accidental because emissivity of unpaired quark matter depends on the value we assumed for the strong coupling constant. From Fig. 1 we see that the temperature of stars with a LOFF core drops faster than ordinary neutron stars. Some interesting phenomenological consequences could exist (for compilation of data and comparison between theoretical models and data see, e.g. 13]). Slow cooling is typical of stars made of ordinary nuclear matter or of stars with a CSC quark core in a gapped phase (e.g. CFL). If a careful comparison with the data could allow to rule out slow cooling for star masses, in the range we have considered, this would favor either the presence of condensed mesons or quark matter in one of the gapless states in the core. Among them the LOFF state is favored since we know that other phases with homogeneous gap parameters such as, e.g. the gCFL or the g2SC phase are instable [14]. Let us finally observe that our results should be considered as preliminary, since the simple ansatz (11) should be substituted by a more complex structure as in [15]. Notwithstanding this question, our conclusions should remain valid, at least qualitatively, also for more complex crystalline patterns of the LOFF condensate. The existence of gapless points and of blocking regions in momentum space characterize the scaling laws for neutrino emissivity and specific heat. Since these properties are typical of the LOFF state, independently of the detailed form of the condensate, a rapid cooling should be appropriate not only for the simple ansatz we assumed, but, more generally, for any LOFF condensate.

I would like to thank D. Blaschke and BLTP-JINR for the pleasant organization of DM2006 school and for kind hospitality; M. Ruggieri, G. Nardulli and M. Mannarelli for the fruitful collaboration which has yielded the work [12] whose results underlie the present paper; N. Ippolito, I. Parenti, M. Buballa and H. Grigorian for useful discussions during the school.
[1] S. L. Shapiro and S. A. Teukolsky, Black Holes, White Dwarfs and Neutron Stars, (New York: Wiley, 1983).

[2] J. C. Collins and M. J. Perry, Phys. Rev. Lett. 34, 1353 (1975)

[3] N. Iwamoto, Phys. Rev. Lett. 44, 1637 (1980); Ann. Phys. 141, 1 (1982).

[4] M. G. Alford, K. Rajagopal and F. Wilczek, Phys. Lett. B 422, 247 (1998); R. Rapp, T. Schäfer, E. V. Shuryak and M. Velkovsky, Phys. Rev. Lett. 81, 53 (1998); R. D. Pisarski and D. H. Rischke, Phys. Rev. D 61, 074017 (2000); M. G. Alford, K. Rajagopal and F. Wilczek, Nucl. Phys. B 537, 443 (1999); K. Rajagopal and F. Wilczek, hep-ph/0011333 M. G. Alford, Ann. Rev. Nucl. Part. Sci. 51, 131 (2001); G. Nardulli, Riv. Nuovo Cim. 25N3, 1 (2002); M. Buballa, Phys. Rept. 407, 205 (2005).

[5] M. Alford and K. Rajagopal, hep-ph/0606157
[6] I. Shovkovy and M. Huang, Phys. Lett. B 564, 205 (2003) arXiv:hep-ph/0302142; M. Alford, C. Kouvaris and K. Rajagopal, Phys. Rev. D 71, 054009 (2005).

[7] A. I. Larkin and Yu. N. Ovchinnikov, Zh. Eksp. Teor. Fiz. 47, 1136 (1964); P. Fulde and R. A. Ferrell, Phys. Rev. 135, A550 (1964); M. G. Alford, J. A. Bowers and K. Rajagopal, Phys. Rev. D 63, 074016 (2001); R. Casalbuoni and G. Nardulli, Rev. Mod. Phys. 76, 263 (2004).

[8] M. Ciminale, G. Nardulli, M. Ruggieri and R. Gatto, Phys. Lett. B 636, 317 (2006).

[9] R. Casalbuoni, R. Gatto, N. Ippolito, G. Nardulli and M. Ruggieri, Phys. Lett. B 627, 89 (2005) [Erratumibid. B 634, 565 (2006)]; M. Mannarelli, K. Rajagopal and R. Sharma, Phys. Rev. D 73, 114012 (2006) arXiv:hep-ph/0603076.

[10] M. Alford, P. Jotwani, C. Kouvaris, J. Kundu and K. Ra- 
jagopal, Phys. Rev. D 71, 114011 (2005).

[11] P. Jaikumar, C. D. Roberts and A. Sedrakian, Phys. Rev. C 73, 042801 (2006) arXiv:nucl-th/0509093; A. Schmitt, I. A. Shovkovy and Q. Wang, Phys. Rev. D 73, 034012 (2006) arXiv:hep-ph/0510347; Q. Wang, Z. g. Wang and J. Wu, Phys. Rev. D 74, 014021 (2006) arXiv:hep-ph/0605092 .

[12] R. Anglani, G. Nardulli, M. Ruggieri and M. Mannarelli, Phys. Rev. D 74, 074005 (2006)

[13] D. Blaschke, H. Grigorian and D. N. Voskresensky, Astron. Astrophys. 368, $561 \quad$ (2001) arXiv:astro-ph/0009120; D. Page, M. Prakash,
J. M. Lattimer and A. Steiner, Phys. Rev. Lett. 85, 2048 (2000); T. Klahn et al., arXiv:nucl-th/0609067.

[14] M. Huang and I. A. Shovkovy, Phys. Rev. D 70, 051501 (2004); R. Casalbuoni, R. Gatto, M. Mannarelli, G. Nardulli and M. Ruggieri, Phys. Lett. B 605, 362 (2005) [Erratum-ibid. B 615, 297 (2005)]; K. Fukushima, Phys. Rev. D 72, 074002 (2005); M. Alford and Q. h. Wang, J. Phys. G 31, 719 (2005).

[15] K. Rajagopal and R. Sharma, arXiv:hep-ph/0605316; K. Rajagopal and R. Sharma, arXiv:hep-ph/0606066. 